Pacific Journal of 


\title{
ON THE EXCEPTIONAL SETS FOR SPACES OF POTENTIALS
}

\author{
DAVID R. ADAMS
}

\begin{abstract}
New results on the Bessel and Besov-Lipschitz potentials on $\boldsymbol{R}^{n}$ are obtained via recent results in nonlinear potential theory. In particular their respective exceptional classes are shown to be identical when $p>2-\alpha / n$. By the same techniques, results on thin sets and traces of potentials are obtained.
\end{abstract}

1. Introduction. In the theory of "perfect functional completion" of a given normed linear space of smooth functions defined on $\boldsymbol{R}^{n}$, the idea is to look for a Banach space with respect to the given norm in, say, the class of Lebesgue measurable functions by taking limits in the norm of smooth functions. Associated in a natural way with any such completion is a $\sigma$-algebra of exceptional sets of $\boldsymbol{R}^{n}$. These exceptional sets give the limits up to which one can pick a canonical equivalence class representative that is defined on the largest possible set. In this note, the exceptional sets for two important perfect functional completions are reexamined in light of recent development in nonlinear potential theory - see e.g., [3], [4], and [7]. The two classes of interest are: $\Lambda_{\alpha, p}=\Lambda_{\alpha p}\left(\boldsymbol{R}^{n}\right)$, the BesovLipschitz potentials on $\boldsymbol{R}^{n}$, and $L_{\alpha, p}=L_{\alpha p}\left(\boldsymbol{R}^{n}\right)$, the Bessel potentials on $\boldsymbol{R}^{n}$. Their respective exceptional classes are denoted by $\mathfrak{X}^{\alpha, p}$ and $\mathfrak{B}^{\alpha, p}$ in [5], where they are studied extensively - see especially Chapter III page 289 in [5] where a criterion for belonging to $\mathfrak{2}^{\alpha, p}$ or $\mathfrak{B}^{\alpha, p}$ is given. This is utilized in Proposition 1 below.

$L_{\alpha, p}\left(\boldsymbol{R}^{n}\right)=g_{\alpha}\left(L_{p}\left(\boldsymbol{R}^{n}\right)\right)$, i.e., the convolution image of the $p$-summable functions on $\boldsymbol{R}^{n}, 1 \leqq p \leqq \infty$, under the Bessel kernel $g_{\alpha}=g_{\alpha}^{(n)}(x)$, the $L_{1}\left(\boldsymbol{R}^{n}\right)$ function whose Fourier transform is $\left(1+|\xi|^{2}\right)^{-\alpha / 2}, \xi \in \boldsymbol{R}^{n}$, $\alpha>0$. The norm on $L_{\alpha . p}$ is $\|u\|_{\alpha p}=\|f\|_{p}$, where $u=g_{\alpha} * f\left(\|\cdot\|_{p}\right.$ the usual norm on $L_{p}$ ). For $\Lambda_{\alpha, p}$, we say $u \in \Lambda_{\alpha p}, 1 \leqq p \leqq \infty, 0<$ $\alpha<1$, if $u \in L_{p}$ and

$$
|u|_{\alpha, p} \equiv\|u\|_{p}+\left\{\int_{R^{n}} \int_{R^{n}}\left(\frac{\left|\Delta_{y} u(x)\right|}{|y|^{\alpha}}\right)^{p} \frac{d x d y}{|y|^{n}}\right\}^{1 / p}
$$

is finite, $\Delta_{y} u(x)=u(x-y)-u(x)$. For $1 \leqq \alpha<2, \Delta_{y} u(x)$ is replaced by $\Delta_{y}^{2} u(x)=u(x-y)+u(x+y)-2 u(x)$ in (1). And finally for $\alpha \geqq 2$, $u \in \Lambda_{\alpha, p}$ iff $u \in L_{p}$ and $\partial u / \partial x_{k} \in \Lambda_{\alpha-1, p}, k=1, \cdots, n$. Other equivalent definitions of $\Lambda_{\alpha, p}$ can be found in [9]. 
It is well known that $\Lambda_{\alpha, 2}=L_{\alpha, 2}$ for all $\alpha>0$ and hence $\mathfrak{2}^{\alpha, 2}=$ $\mathfrak{B}^{\alpha, 2}$. But for $1 \leqq p \leqq 2, \Lambda_{\alpha, p} \subset L_{\alpha, p}$ and for $2 \leqq p \leqq \infty, L_{\alpha, p} \subset \Lambda_{\alpha, p}$. Moreover, these inclusion are proper for $p \neq 2$. (See [9].) Thus, although the classes $\Lambda_{\alpha, p}$ and $L_{\alpha, p}$ are quite different in many respects it can be shown (via nonlinear potential theory) that $\mathfrak{2}^{\alpha, p}=\mathfrak{B}^{\alpha, p}$, $\alpha>0,2-\alpha / n<p<\infty$. When $1 \leqq p \leqq 2-\alpha / n$, the result remains open. It is this and related results that are discussed here.

2. Main result. Consider the following set functions (capacities) defined initially for compact set $K \subset \boldsymbol{R}^{n}$ :

$$
B_{\alpha, p}^{(n)}(K)=\inf \|\varphi\|_{\alpha, p}^{p}
$$

and

$$
A_{\alpha, p}^{(n)}(K)=\inf |\varphi|_{\alpha, p}^{p}
$$

where, in each case, the infimum is over $\varphi \in C_{0}^{\infty}\left(\boldsymbol{R}^{n}\right)$ for which $\varphi(x) \geqq 1$ on $K . C_{0}^{\infty}\left(\boldsymbol{R}^{n}\right)$ denotes the infinitely differentiable functions on $\boldsymbol{R}^{n}$ with compact support.

REMARK. $B_{\alpha, p}^{(n)}$ and $A_{\alpha, p}^{(n)}$ can be extended to all sets of $\boldsymbol{R}^{n}$ as "outer capacities" - see e.g., [7].

Proposition 1. $A_{\alpha, p}^{(n)}(K)=0\left(B_{\alpha, p}^{(n)}(K)=0\right)$ iff $K \in \mathfrak{Q}^{\alpha, p}\left(K \in \mathfrak{B}^{\alpha, p}\right), K$ a compact set of $\boldsymbol{R}^{n}$.

THEOREM 1. $A_{\alpha, p}^{(n)}(K)=0$ iff $B_{\alpha, p}^{(n)}(K)=0, \alpha>0,2-\alpha / n<p<\infty$, $K$ a compact set of $\boldsymbol{R}^{n}$, and for $\alpha p>1, p>1$ if $K$ is a compact subset of $\boldsymbol{R}^{n-1}$.

For the proof, we need to draw from two sources - the key facts are Theorems I and II below.

THEOREM I ([8]). If for $\varphi \in C_{0}^{\infty}\left(\boldsymbol{R}^{n}\right), R_{m} \varphi=\varphi\left(x_{1}, \cdots, x_{m}, 0, \cdots, 0\right)$, $1 \leqq m \leqq n$, then there exists a linear extension operator $E_{m}$ such that for $\psi \in C_{0}^{\infty}\left(\boldsymbol{R}^{m}\right), R_{m}\left(E_{m} \psi\right)=\psi$. Furthermore, there is a constant $C$ independent of $\varphi$ and $\psi$ such that

(a) $\left|R_{m} \varphi\right|_{\beta, p} \leqq C\|\varphi\|_{\alpha, p}$

(b) $\left\|E_{m} \varphi\right\|_{\alpha, p} \leqq C|\psi|_{\beta, p}$

(c) $\left|R_{m} \varphi\right|_{\beta, p} \leqq C|\varphi|_{\alpha, p}$

(d) $\left|E_{m} \psi\right|_{\alpha, p} \leqq C|\psi|_{\beta, p}$

whenever $1<p<\infty, \beta=\alpha-(n-m / p)>0$.

Theorem II ([2]). For a Borel measure $\mu$, set

$$
U_{\alpha, p}^{(n)}(x)=g_{\alpha}^{(n)} *\left(g_{\alpha}^{(n)} * \mu\right)^{1 /(p-1)}(x),
$$


then

$$
U_{\alpha, p}^{(n)}(x) \sim \int_{0}^{\infty}\left[r^{\alpha p-n} \mu\left(S_{r}(x)\right)\right]^{1 /(p-1)} e^{-b r} \frac{d r}{r}
$$

for $p>2-\alpha / n, 0<\alpha p \leqq n$. The symbol $\sim$ means that the ratio is bounded above (for some $b>0$ ) and below (for another $b>0$ ), the bounds being independent of $x$ and $\mu . \quad S_{r}(x)=$ ball of radius $r$ about $x \in R^{n}$.

Now by $I(a), A_{\beta, p}^{(m)}(K) \leqq C B_{\alpha, p}^{(n)}(K)$ for some $C>0$ independent of $K \subset \boldsymbol{R}^{m}$ since the restriction $R_{m}$ of each test function for $B$ is a test function for $A$. Similarly, $B_{\alpha, p}^{(n)}(K) \leqq C A_{\beta, p}^{(m)}(K)$ using $I(b)$. Hence, $I(a-d)$ implies that $B_{\alpha, p}^{(n)} \sim A_{\beta, p}^{(m)} \sim A_{\alpha, p}^{(n)}$, on compact subsets of $\boldsymbol{R}^{m}$, $m \leqq n-1$. To remove this restriction on $K$, we use II. By [7] we know that for any compact $K \subset \boldsymbol{R}^{m}, B_{\alpha, p}^{(n)}(K)>0$ iff there is a nonzero Borel measure concentrated on $K$ such that $U_{\alpha, p}^{(n)}$ is bounded. But since $\alpha p-n=\beta p-m$, II gives $B_{\beta, p}^{(m)}(K)>0$ iff $B_{\alpha, p}^{(n)}(K)>0$, $p>2-\beta / m, 0<\alpha p \leqq n$. (This relation trivially holds when $\alpha p>n$.) It might be noted that " $B_{\alpha, p}^{(n)}(K)=0$ implies $B_{\beta, p}^{(m)}(K)=0$ for $1<p<\infty$ " is an immediate consequence of the definition of Bessel capacity - a fact we have improved for $p>2-\beta / m$.

Note, if we change the notation slightly in the above arguments, we have: $B_{\alpha, p}^{(n)}(K)=0$ iff $B_{\alpha+k \mid p, p}^{(n+k)}(K)=0, p>2-\alpha / n, K$ compact in $\boldsymbol{R}^{n}, k$ a positive integer.

Corollary 1. $B_{\alpha, p}^{(n)}(K)=0$ iff $B_{\beta, p}^{(m)}(K)=0, \beta=\alpha-(n-m) / p>$ $0, p>2-\beta / m, K$ compact in $\boldsymbol{R}^{m}$.

3. Thin sets. A set $E \subset R^{n}$ is called $\mathscr{F}_{\alpha, p}^{(n)}$ - thin at $x_{0} \in \bar{E}$ iff there exists a Borel measure $\mu$ such that $U_{\alpha, p}^{(n)}(x)$ is bounded and

$$
U_{\alpha, p}^{(n)}(x)<\lim _{\substack{x \rightarrow x_{0} \\ x \in E}} \inf U_{\alpha, p}^{(n)}(x)
$$

Recently in [4], necessary and sufficient conditions of the Wiener type have been given for a set to be $\mathscr{F}_{\alpha, p}^{(n)}-$ thin at $x_{0}$, provided $p>2-$ $\alpha / n$. The condition, which depends strongly on II, is by [4] and Theorem 1

$$
\int_{0}^{1}\left[r^{\alpha p-n} A_{\alpha, p}^{(n)}\left(E \cap S_{r}\left(x_{0}\right)\right)\right]^{1 /(p-1)} \frac{d r}{r}<\infty
$$

It was also shown in [4] that (3) is not equivalent to $\mathscr{F}_{\alpha, p}^{(n)}-$ - thinness for $1<p<2-\alpha / n$. So, although it remains unknown as to 
what the appropriate replacement for (3) is when $p<2-\alpha / n$, it is of interest to know just what (3) means when $p \leqq 2-\alpha / n$. In this vain, Theorem 1 gives

Theorem 2. If $E \subset \boldsymbol{R}^{n}$, then (3) is equivalent to $E$ being $\mathscr{F}_{\alpha+k \mid p, p}^{(n+k)}-$ thin at $x_{0}$. Here $k$ is a positive integer chosen large enough so that $p>1+0(1 / \sqrt{k})$. Furthermore, $k$ can be chosen to be zero provided $p>2-\alpha / n$.

4. Traces of $\Lambda_{\alpha, p}$ - potentials. The techniques of Theorem 1 can also be used to obtain trace inequalities in the spirit of [1] and [2]. If $\Phi(\cdot, \nu)$ is a semi-norm on $C_{0}^{\infty}\left(\boldsymbol{R}^{n}\right)$ for each Borel measure $\nu$, such that $\Phi(\phi, \nu)=0$ when $\phi$ is zero on the support of $\nu$, then

THEOREM 3. If for any $\alpha>0$ and $1<p<\infty$, there is a constant $C$ independent of $u \in C_{0}^{\infty}\left(\boldsymbol{R}^{n}\right)$ such that

$$
\Phi(|u|, \nu) \leqq C\|u\|_{\alpha, p}
$$

then

$$
\Phi(|u|, \nu) \leqq C^{\prime}|u|_{\alpha, p}
$$

for some constant $C^{\prime}$ independent of $u$. The converse holds for $p \geqq 2$.

For various choices of $\Phi$ we can obtain trace inequalities for the $\Lambda$-spaces analogous to those given in [1] and [2] for the $L_{\alpha, p}$-spaces. In particular, when $\Phi$ is a Lorentz norm, we get Sobolev type inequalities for the $\Lambda$-spaces from the known inequalities for the Bessel potentials (cf. [6]). Thus from [2] we have the following rather interesting

CoRollary 2. Suppose $\mu$ is a Borel measure on $\boldsymbol{R}^{n}$ with compact support such that for all $x \in \boldsymbol{R}^{n}$ and $r>0$, and any $d: 0<d \leqq n$, $\mu\left(S_{r}(x)\right) \leqq C r^{d}$, then for $\alpha p=n, 1<p<\infty$, and some $b>0$,

$$
\sup _{|u|_{\alpha, p} \leqq 1} \int \exp \left(b|u(x)|^{p^{\prime}}\right) d \mu(x)<\infty,
$$

$p^{\prime}=p /(p-1)$.

A further application of the techniques of Theorem 1, is to the results of [10], where Fubini type theorems with respect to $A_{\alpha, p}$ and $B_{\alpha, p}$ null sets are discussed. Theorem 1 improves the apparent assymetry in these results. 


\section{REFERENCES}

1. D. R. Adams, Traces of potentials of arising from translation invariant operators, Ann. Sc. Norm. Sup. Pisa., 25 (1971), 203-217.

2. - Traces of potentials. II, Indian Univ. Math. J., 22 (1973), 907-918.

3. D. R. Adams and N. G. Meyers, Bessel potentials Inclusion relations among classes of exceptional sets, Indiana Univ. Math. J., 22 (1973), 873-905.

4. - Thinness and Wiener criteria for nonlinear potentials, Indiana Univ. Math. J., 22 (1972), 169-197.

5. N. Aronszajn, F. Mulla, and P. Szeptycki, On spaces of potentials connected with $L^{p}$ classes, Ann. Inst. Fourier, 13 (1963), 211-306.

6. C. Herz, Lipschitz spaces and Bernstein's theorem on absolutely convergent Fourier transforms, J. Math. Mech., 18 (1968), 283-323.

7. N. G. Meyers, A theory of capacities for potentials of functions in Lebesgue classes, Math. Scand., 26 (1970), 255-292.

8. E. M. Stein, The characterization of functions arising as potentials. II, Bull. Amer. Math. Soc., 68 (1962), 577-582.

9. M. H. Taibleson, On the theory of Lipschitz spaces of distributions on Euclidean n-space. I, J. Math. Mech., 13 (1964), 407-480.

10. P. Szeptycki, On restrictions of functions in the spaces $P^{\alpha, p}$ and $B^{\alpha, p}$, Proc. Amer. Math. Soc., 16 (1965), 341-347.

Received August 17, 1973. The research was partially supported by NSF GP-33749.

University of California, San Diego

AND

UNIVERSITY OF KeNTUCKY 



\section{PACIFIC JOURNAL OF MATHEMATICS}

EDITORS

RICHARD ARENS (Managing Editor)

University of California

Los Angeles, California 90024

R. A. Beaumont

University of Washington

Seattle, Washington 98105

J. DugundJI

Department of Mathematics

University of Southern California

Los Angeles, California 90007

D. Gilbarg and J. Milgram

Stanford University

Stanford, California 94305

\section{ASSOCIATE EDITORS}

E. F. BECKENBACH

B. H. NeumanN

F. WOLF

K. YOSHIDA

\section{SUPPORTING INSTITUTIONS}

UNIVERSITY OF BRITISH COLUMBIA CALIFORNIA INSTITUTE OF TECHNOLOGY

UNIVERSITY OF CALIFORNIA

MONTANA STATE UNIVERSITY

UNIVERSITY OF NEVADA

NEW MEXICO STATE UNIVERSITY

OREGON STATE UNIVERSITY

UNIVERSITY OF OREGON

OSAKA UNIVERSITY

\author{
UNIVERSITY OF SOUTHERN CALIFORNIA \\ STANFORD UNIVERSITY \\ UNIVERSITY OF TOKYO \\ UNIVERSITY OF UTAH \\ WASHINGTON STATE UNIVERSITY \\ UNIVERSITY OF WASHINGTON \\ $\stackrel{*}{*} \stackrel{*}{*}{ }^{*}{ }^{*}{ }^{2}$ AMERICAN MATHEMATICAL SOCIETY \\ NAVAL WEAPONS CENTER
}




\section{Pacific Journal of Mathematics}

\section{Vol. 52, No. $1 \quad$ January, 1974}

David R. Adams, On the exceptional sets for spaces of potentials ............ 1

Philip Bacon, Axioms for the Čech cohomology of paracompacta ............ 7

Selwyn Ross Caradus, Perturbation theory for generalized Fredholm operators ..... 11

Kuang-Ho Chen, Phragmén-Lindelöf type theorems for a system of nonhomogeneous equations ............................ 17

Frederick Knowles Dashiell, Jr., Isomorphism problems for the Baire classes .......

M. G. Deshpande and V. K. Deshpande, Rings whose proper homomorphic images are right subdirectly irreducible . . . . . . . . . . . . . . . . . . . . . . . . .

Mary Rodriguez Embry, Self adjoint strictly cyclic operator algebras .............

Paul Erdős, On the distribution of numbers of the form $\sigma(n) / n$ and on some related

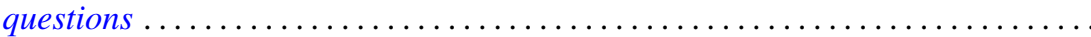

Richard Joseph Fleming and James E. Jamison, Hermitian and adjoint abelian

operators on certain Banach spaces ............................

Stanley P. Gudder and L. Haskins, The center of a poset .................. 85

Richard Howard Herman, Automorphism groups of operator algebras . . . ........

Worthen N. Hunsacker and Somashekhar Amrith Naimpally, Local compactness of families of continuous point-compact relations ....................

Donald Gordon James, On the normal subgroups of integral orthogonal groups ....

Eugene Carlyle Johnsen and Thomas Frederick Storer, Combinatorial structures in

loops. II. Commutative inverse property cyclic neofields of prime-power

order.

Ka-Sing Lau, Extreme operators on Choquet simplexes . . . . . . . . . . . . . . 129

Philip A. Leonard and Kenneth S. Williams, The septic character of 2, 3, 5 and $7 \ldots 143$

Dennis McGavran and Jingyal Pak, On the Nielsen number of a fiber map ........ 149

Stuart Edward Mills, Normed Köthe spaces as intermediate spaces of $L_{1}$ and

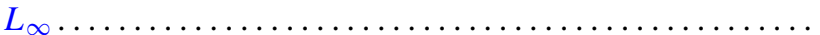

Philip Olin, Free products and elementary equivalence. .

Louis Jackson Ratliff, Jr., Locally quasi-unmixed Noetherian rings and ideals of the principal class.

Seiya Sasao, Homotopy types of spherical fibre spaces over spheres ...

Helga Schirmer, Fixed point sets of polyhedra ...

Kevin James Sharpe, Compatible topologies and continuous irreducible

representations.

Frank Siwiec, On defining a space by a weak base . . . . . . . . . . . . . . . 233

James McLean Sloss, Global reflection for a class of simple closed curves ....... 247

M. V. Subba Rao, On two congruences for primality . .

Raymond D. Terry, Oscillatory properties of a delay differential equation of even

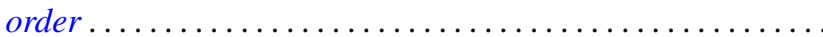

Joseph Dinneen Ward, Chebyshev centers in spaces of continuous functions . .

Robert Breckenridge Warfield, Jr., The uniqueness of elongations of Abelian

groups...

V. M. Warfield, Existence and adjoint theorems for linear stochastic differential

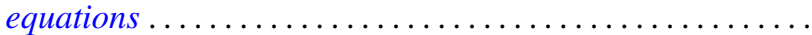

